\title{
The Concept of the Auditory Training for Blind and Visually Impaired Children and Teenagers
}

\author{
E. Skrodzka*, M. Maciągowski and A. Furmann \\ Institute of Acoustics, A. Mickiewicz University \\ Umultowska 85, 61-614 Poznań, Poland
}

\begin{abstract}
The paper was inspired by the hitherto published results concerning provision of efficient assistance to visually impaired persons by means of acoustic and vibratory signals. The aim of the auditory training is to shorten the time necessary for execution of auditory information processing, sensibilize blind persons to differences in sounds and teach them to focus auditory attention on small differences in parameters of acoustic waves, in order to achieve independent and correct interpretation of environment by hearing and listening. The basic concept of the auditory training addressed to blind or visually impaired children and teenagers is presented in detail. Preliminary verification of the effectiveness of training of young adults without vision impairments has not shown statistically significant differences in performance before and after the training. Possible reasons for such an effect are pointed out and solutions are suggested.
\end{abstract}

DOI: 10.12693/APhysPolA.125.A-31

PACS: 43.66.Yw, 43.66.Hg, 43.66.Jw, 43.66.Qp

\section{Introduction}

Reception and correct interpretation of acoustically conveyed information is very important for blind and visually impaired children and adolescents for safe and effective navigation in the environment and protection against social exclusion. A set of characteristic features of a sound source is encoded in physical parameters of the sound it generates. The features permit distinction of different sound sources and evaluation of changing acoustic situation.

The aim of the work is preparation of an auditory training (AT) comprising selected psychoacoustic and lateralization tasks for blind and visually impaired children and adolescents. Psychoacoustic tasks are related to changes in basic sound wave parameters during its propagation in the environment. The lateralization tasks test the basic auditory skill of the blind and teach proper orientation on a crossroad or recognition of direction of an approaching motor vehicle. The aim of AT is to shorten the time necessary for execution of the auditory information processing, sensibilize the blind persons to differences in sounds, and teach them to focus auditory attention on small differences in parameters of an acoustic wave which is essential for independent and correct interpretation of environment by hearing and listening. The work was inspired by results of hitherto projects related to provision of assistance to visually impaired persons by means of acoustic and vibratory signals [1-10].

There are reports showing that blind people perform much better than normally sighted individuals in the tasks involving concentration and localization, speech perception, memorization of words or discrimination of

*corresponding author; e-mail: af a@amu.edu.pl pitch [11-16]. It is suggested that people who are congenitally blind or who have lost their sight at an early age are better at identifying and differentiating sounds than those who have become blind in adolescence or adulthood [16-17]. The studies concerning perception of sounds and vibrations by the blind have been generally performed in the adult population. Reports of equivalent or similar experiments carried out with blind children/adolescents are very scarce [2,5-7]. The research concerning the effect of acoustic training on spatial updating in congenitally blind adults has shown that the accuracy of verbal estimates of the magnitude of distance has been improved after the training [18]. The report describing the influence of early non-visual experience on the acuity of proprioceptive-spatial discrimination has shown that congenitally blind adults who attended orientation and mobility training before the age of 12 years, compensated the lack of vision by hearing and touch better than people who started training after the age of 12 [19]. Speech-in-noise intelligibility in the older blind frequently listening to recorded speech material (e.g. audiobooks) was better than in the reference group of sighted participants who had not used speaking devices [20], probably due to better concentration on verbal information and listening experience. On the basis of the above information it was expected that a proper AT may be beneficial to the blind/visually impaired people, provided that it would be started at the appropriate moment of life. Taking into account the above short review of research reports we claim that the present paper deals with fundamental and innovatory issue concerning AT addressed to children and adolescents.

\section{Basic concept}

The training is addressed mainly to two groups of blind/visually impaired individuals, aged 8-12 and 1318. The division into age groups is made on the basis of the two facts: 
1) the studies into brain functioning indicate that people who lost vision at an early age display higher activity in occipital parts of the brain during tasks not requiring vision than individuals who became blind at a later age in life [21-22]. The critical age is $12-14$ years. People with congenital blindness or loss of vision occurring in childhood are more prone to changes in brain functioning and its intra- and intermodal plasticity induced by their condition,

2) preliminary studies have shown differences between blind 10- and 15-year-olds in the accuracy of performing tasks involving pitch discrimination, and pitch and timbre discrimination. Results obtained for 10-year-olds with visual dysfunctions did not differ statistically from those recorded in the sighted reference group. However, visually dysfunctional 15-year-olds scored much better than blind 10-year-olds and normally-sighted children and adolescents from the reference group $[2,6]$.

Each training session was preceded by a short preliminary session to make the listeners familiar with the task and clarify any doubts or concerns. In four weeks a blind/visually impaired participant was trained in 20 training session, each lasting for about 24 minutes (depending on a difficulty level of presented tasks). Training sessions were as short as possible because of participation of young children. The lateralization tasks involved binaural audio reproduction via headphones, while the remaining experiments were based on monaural audio reproduction using a single headphone in the preferred ear. The sequence of tasks and the sequence of signals in the pair of signals was random. The effect of AT on performance of the blind or visually impaired subjects was evaluated on the basis of statistical analysis of differences in results obtained in pre-and post-test (collected before and immediately after training).

\section{Auditory training content}

The auditory training was prepared on PC-class workstation with Magix Samplitude software package. Open headphones Sennheiser HD600 together with headphone distributor Head Acoustics PEQ IV were used for presentation of sounds. The sounds presented during the training were prepared in MATLAB environment and presented at a comfortable level of $65 \mathrm{~dB}$ SPL. Each session was divided into two parts, and listeners could take a break between them. Table I presents a description of tasks performed during the training. Apart from the category "miscellaneous", performance of all tasks was scored and with the progress in the listener, the level of difficulty $(n)$ increases. Each session comprised 14 tasks. Performance of a given task was approved when the listener achieved $90 \%$ of correct responses. After successful completion of one session, the listener was asked to start the next one of a higher level of difficulty. The level of difficulty of particular tasks could be different depending on the progress of the subject, but the subject was not informed about the progress he or she has made. If not specified otherwise, the stimulus duration was $1 \mathrm{~s}$, and the break between two stimuli in a pair was $1 \mathrm{~s}$. The standard time to give the response was $4 \mathrm{~s}$ but it could be longer.

General information about the auditory training.

TABLE I

\begin{tabular}{l|l}
\hline \hline Auditory training, part 1 & Auditory training, part 2 \\
\hline \hline $\begin{array}{l}\text { 1. Pitch discrimination } \\
\text { - tones }\end{array}$ & $\begin{array}{l}\text { 1. Loudness discrimination } \\
\text { - tones }\end{array}$ \\
\hline $\begin{array}{l}\text { 2. Pitch discrimination } \\
\text { - noise bands }\end{array}$ & $\begin{array}{l}\text { 2. Loudness discrimination } \\
\text { - noise bands }\end{array}$ \\
\hline 3. Pitch discrimination & $\begin{array}{l}\text { 3. Loudness discrimination } \\
\text { - FM }\end{array}$ \\
\hline $\begin{array}{l}\text { 4. Lateralization of sound } \\
\text { - tones }\end{array}$ & $\begin{array}{l}\text { 4. Lateralization of sound } \\
\text { - noise }\end{array}$ \\
\hline $\begin{array}{l}\text { 5. Pitch-timbre categoriza- } \\
\text { tion - musical instruments }\end{array}$ & \begin{tabular}{l} 
5. Pitch memory \\
\hline \multicolumn{2}{c}{ Timbre discrimination - harmonic signals }
\end{tabular} \\
\hline 8. Signal-in-noise detection & 8. Miscellaneous \\
\hline 9. Miscellaneous &
\end{tabular}

The division of time among particular types of tasks (related to particular features of acoustic signals) in the whole training was as follows: timbre discrimination $44 \%$ of total time; pitch discrimination - $14 \%$; loudness discrimination - 14\%; sound source lateralization - $9 \%$; pitch memory - 6\%; signal-in-noise detection - 3\%; miscellaneous $-9 \%$. The time allowed for realization of particular tasks was established on the basis of literature $[2,6,23]$ and a preliminary experiment carried out with a group of children. The most serious problems were encountered in correct performance of the tasks related to timbre discrimination, in particular when the listeners were asked to discriminate simultaneously the timbre and pitch of the sound. The notion of timbre was difficult to be intuitively grasped, so much time and attention were dedicated to the tasks related to this property of sound. The easiest tasks proved to be those related to signal detection against the background noise, so the least time was devoted to the relevant tasks. The training was addressed also to children so the tasks were versatile and short, not to bore the participants. The interval between the easiest and the most difficult levels was divided into a few or over a dozen sublevels of intermediate difficulty. The level of difficulty was increased with the listener's progress by reducing the difference between the stimuli.

\subsection{Pitch discrimination - tones and pitch discrimination - noise bands (AT, part 1)}

The above-mentioned two types of tasks were based on the results reported in [2]. Pairs of tonal sounds of different frequencies and pairs of $1 / 3$-octave noise bands of different center frequencies were used. The listeners were asked to point out a higher sound in the pair. The range of frequency of the stimuli was 100-4000 Hz. In Table II are presented the detuning of the frequencies of both tonal signals (tones discrimination)and the detuning of the center frequency and signal duration (noise bands discrimination) for different levels of difficulty. The center frequency range of noise stimuli was from the range $125-2500 \mathrm{~Hz}$. 
TABLE II

Signal parameters for pitch discrimination of: tones (frequency difference (1)) and noise bands (center frequency difference (2) and time duration of a single noise signal (3)) for different levels of difficulty.

\begin{tabular}{c|c|cc}
\hline \hline \multirow{2}{*}{$n$} & tones & \multicolumn{2}{|c}{ noise bands } \\
\cline { 2 - 4 } & $(1)$ & $(2)$ & $(3)$ \\
\hline 1 & $1 / 3$-octave & $1 / 3$-octave & $1 \mathrm{~s}$ \\
\hline 2 & $5 \%$ & $10 \%$ & $1 \mathrm{~s}$ \\
\hline 3 & $3 \%$ & $8 \%$ & $1 \mathrm{~s}$ \\
\hline 4 & $2 \%$ & $5 \%$ & $1 \mathrm{~s}$ \\
\hline 5 & $1.5 \%$ & $3 \%$ & $1 \mathrm{~s}$ \\
\hline 6 & $1 \%$ & $2 \%$ & $1 \mathrm{~s}$ \\
\hline 7 & $0.75 \%$ & $1 \%$ & $1 \mathrm{~s}$ \\
\hline 8 & $0.5 \%$ & $1 \%$ & $0.3 \mathrm{~s}$ \\
\hline 9 & $0.25 \%$ & &
\end{tabular}

\subsection{Pitch-timbre categorization - musical instruments (AT, part 1)}

The aim of the exercise was to acquaint the subject with simultaneous discrimination of pitch and timbre and to introduce him/her to the pitch timbre categorization of the harmonic signals task. Pairs of musical instrument sounds differing in spectral content and fundamental frequency were presented to a subject. The subject was asked to identify the difference between the sounds presented. The possible answers were 'timbre', 'pitch' or 'both features'. The sounds were those emitted by musical instruments (piano, guitar, vibraphone, organ) of the fundamental frequencies and the corresponding pitch as shown in Table III, the differences in the fundamental frequencies always corresponded to the major third. If the difference in pitch was considered, the sounds presented were generated on the same instrument. If the difference in timbre was to be recognized, the two sounds of the same pitch were generated by different instruments. If the differences in both pitch and timbre had to be pointed out, the two sounds of different pitch were generated by two different instruments. The listener was asked to perform tasks at the same level of difficulty as long as he/ she has reached $100 \%$ of correct responses.

TABLE III

Fundamental frequencies [Hz] (1) of sounds (2) in pitch for timbre categorization - musical instruments task.

\begin{tabular}{l|c|c|c|c|c|c|c|c|c|c}
\hline \hline$(1)$ & 261.6 & 329.6 & 415.3 & 523.3 & 659.3 & 830.6 & 1046.5 & 1318.5 & 1661.2 & 2093.0 \\
\hline
\end{tabular} \begin{tabular}{l|l|l|l|l|l|l|l|l|l|l}
\hline$(2)$ & $C_{4}$ & $E_{4}$ & $G_{4}^{\# 1}$ & $C_{5}$ & $E_{5}$ & $G_{5}^{\#}$ & $C_{6}$ & $E_{6}$ & $G_{6}^{\#}$ & $C_{7}$
\end{tabular}

3.3. Pitch - timbre categorization - harmonic signals (AT, parts 1 and 2)

The listeners were exposed to pairs of harmonic multitones of different spectral content and fundamental frequency. The fundamental frequency was chosen at random from the range $100-2000 \mathrm{~Hz}$. When the multitones in a given pair differed also in the fundamental frequency, in one of the signals it was detuned towards higher frequencies by the earlier defined value depending on the level of difficulty. The spectral composition of the multitones (when they differed in timbre or timbre and pitch) and the differences in the fundamental frequency (when they differ in pitch or pitch and timbre) for different levels of difficulty are presented in Table IV. The signals could differ in timbre, in pitch or in both these features simultaneously. The subjects were asked to indicate the difference between the sounds presented. When the signals in a given pair differed in pitch, the multitones had different frequencies and the same spectral content. When the signals differed in timbre, the multitones had the same fundamental frequency and different content of harmonic components. When the signals differed in pitch and in timbre, the subject heard two multitones of different fundamental frequency and different content of harmonic components. The idea of tasks 3.3 and 3.4 was inspired by [16].

TABLE IV

Parameters of harmonic signals for timbre/timbre and pitch tasks: number of harmonics (1) and frequency deviation (2).

\begin{tabular}{c|c|c|c|c|c}
\hline \hline$n$ & 1 & 2 & 3 & 4 & 5 \\
\hline$(1)$ & 6 & 4 & 2 & 2 & 2 \\
\hline$(2)$ & $1 / 3$-octave & $10 \%$ & $3 \%$ & $2 \%$ & $1 \%$
\end{tabular}

3.4. Pitch memory (AT, part 2)

The aim of the exercise was to train the short-time memory. The subjects were exposed to sequences of tones of different frequencies corresponding to the pitch of sounds on the musical scale distanced by the interval of a minor second. The frequencies of particular tones in a sequence were selected at random from the set of 10 displayed in Table $\mathrm{V}$. The same table presents also the sound pitch corresponding to these frequencies. The sound sequences were prepared in two variants. In the first one, the frequency of the first and the last signal in the sequence was different. The choice of the sound frequencies was random, but the first and the last sound could not have the same frequency. In the second variant, the choice of the sound frequencies was random too, but the first and the last sound had the same frequency. The subjects were asked to identify the sequence in which the first and the last sound had the same pitch. The number of sounds in a sequence for level of difficulty $n$ was $n+2(n=1 \div 7)$. The duration of an individual sound was $300 \mathrm{~ms}$, the interval between sounds in a sequence was also $300 \mathrm{~ms}$.

TABLE V

Parameters of harmonic signals for pitch-timbre categorization task: number of harmonics (1) and frequency deviation (2).

\begin{tabular}{l|c|c|c|c|c|c|c|c|c|c}
\hline$(1)$ & 440.0 & 466.2 & 493.9 & 523.3 & 554.4 & 587.3 & 622.3 & 659.3 & 698.5 & 740.0 \\
\hline$(2)$ & $A_{4}$ & $A_{4}^{\#}$ & $B_{4}$ & $C_{5}$ & $C_{5}^{\#}$ & $D_{5}$ & $D_{5}^{\#}$ & $E_{5}$ & $F_{5}$ & $F_{5}$ \\
\hline
\end{tabular}

\begin{tabular}{l|l|l|l|l|l|l|l|l|l|l}
\hline$(2)$ & $A_{4}$ & $A_{4}^{\#}$ & $\mathrm{~B}_{4}$ & $C_{5}$ & $C_{5}^{\#}$ & $D_{5}$ & $D_{5}^{\#}$ & $E_{5}$ & $F_{5}$ & $F_{5}^{\#}$
\end{tabular}

\subsection{Pitch discrimination - FM (AT, part 1) and} loudness discrimination - $A M$ (AT, part 2)

In both cases, one signal in a pair of presented sounds was frequency modulated (FM), and the other - amplitude modulated (AM). The signal duration was $3 \mathrm{~s}$. 
The listener compared the signals of the same carrier frequency corresponding to $1 / 3$-octaves from the range $125-2500 \mathrm{~Hz}$, excluding the frequencies for which the frequency modulation difference limen was lower than the discrimination threshold (assumed as $1 \mathrm{~Hz}$ [24]). When two modulated sequences were presented, one FM and one AM, the listener had to find out whether the change in sound came from changes in frequency or amplitude.

In the case of pitch discrimination, the task of the subject was to point out the FM signal, or in other words, indicate the sound the pitch of which has changed. Sinusoidal modulation frequency both in FM and AM signals was $1 \mathrm{~Hz}$. Frequency deviation of FM sounds and modulation depth of AM sounds, depending on difficulty level, are shown in Table VI. In the case of loudness discrimination, the task was to point out the AM signal. Sinusoidal modulation frequency both in FM and AM signals was 2 $\mathrm{Hz}$. The subjects were asked to identify the sound loudness of which had changed.

TABLE VI

Signal parameters for: frequency discrimination - FM task (1); loudness discrimination - AM task (2).

\begin{tabular}{c|c|c|c|c}
\hline \hline & \multicolumn{2}{|c|}{$(1)$} & \multicolumn{2}{c}{$(2)$} \\
\hline$n$ & $F M i^{*}$ & $A D$ & $A D$ & $F M i^{*}$ \\
\hline 1 & $1 / 20$ & 0.5 & 0.5 & $1 / 40$ \\
2 & $1 / 40$ & 0.5 & 0.2 & $1 / 80$ \\
3 & $1 / 80$ & 0.2 & 0.2 & $1 / 160$ \\
4 & $1 / 100$ & 0.1 & 0.1 & $1 / 160$ \\
5 & $1 / 150$ & 0.1 & 0.1 & $1 / 200$ \\
6 & $1 / 200$ & 0.1 & 0.1 & $1 / 300$ \\
7 & $1 / 200$ & 0.075 & 0.075 & $1 / 400$ \\
8 & $1 / 200$ & 0.05 & 0.075 & $1 / 600$ \\
9 & $1 / 300$ & 0.05 & 0.05 & $1 / 400$ \\
10 & $1 / 450$ & 0.05 & 0.05 & $1 / 800$ \\
11 & $1 / 500$ & 0.05 & & \\
* dependent on carrier frequency
\end{tabular}

\subsection{Loudness discrimination - tones and loudness} discrimination - noise bands (AT, part 2)

Pairs of tones or $1 / 3$-octave noise bands with the same frequency/center frequency differing in sound pressure level were presented. Frequency range was 100-4000 Hz. Frequencies chosen were $1 / 3$-octave distant. The aim of the subject was to point out the louder signal. Differences in $S P L, \Delta_{S P L}$, and time duration $T D$ depending of difficulty level are show in Table VII.

Loudness discrimination - tones and

TABLE VII

loudness discrimination — noise tasks.

\begin{tabular}{c|r|r|r|r|r|r|r|c|c}
\hline \hline$n$ & 1 & 2 & 3 & 4 & 5 & 6 & 7 & 8 & 9 \\
\hline$\Delta_{S P L}[\mathrm{~dB}]$ & -10 & -8 & -6 & -4 & -3 & -2 & -1 & -1 & -0.7 \\
$T D[\mathrm{~s}]$ & 1 & 1 & 1 & 1 & 1 & 1 & 1 & 0.3 & 0.3
\end{tabular}

\subsection{Signal-in-noise detection (AT, part 1)}

The listeners were exposed to pairs of signals of which one contained a multitone (a rectangular signal of the carrier frequency $440 \mathrm{~Hz}\left(f_{1}\right)$ or $\left.880 \mathrm{~Hz}\left(f_{2}\right)\right)$, amplitude of which was modulated by a rectangular signal of the frequency $5 \mathrm{~Hz}$, in the presence of white noise, while the second signal was just white noise alone. The listeners were asked to identify the signal in which they had heard a warning signal. The multitone signal-to-noise ratio $(S N R)$ depends on the task difficulty level of are shown in Table VIII. On each level $S N R[\mathrm{~dB}]$ could take one of 4 values.

TABLE VIII

Parameters of signal-in-noise detection task.

\begin{tabular}{c|c|c|c|c|c|c|c|c|c|c}
\hline \hline$n$ & 1 & 2 & 3 & 4 & 5 & 6 & 7 & 8 & 9 & 10 \\
\hline \multirow{2}{*}{$S R$} & 5 & 0 & -8 & -13 & -15 & -18 & -20 & -23 & -20 & -23 \\
{$[\mathrm{~dB}]$} & 0 & -5 & -10 & -15 & -18 & -20 & -23 & -25 & -23 & -25 \\
& -8 & -10 & -13 & -18 & -20 & -23 & -25 & -27 & -25 & -27 \\
\hline$f$ & $f_{1}$ & $f_{1}$ & $f_{1}$ & $f_{1}$ & $f_{1}$ & $f_{1}$ & $f_{1}$ & $f_{1}$ & $f_{2}$ & $f_{2}$
\end{tabular}

\subsection{Timbre discrimination - harmonic signals (AT, parts 1 and 2)}

This task was aimed at teaching analysis and discrimination of the timbre of sound. The listeners were exposed to pairs of harmonic multitones of different spectral contents. The sounds had the same fundamental frequency and different numbers of harmonic components, ranging from 2 to 8 . The fundamental frequency was chosen at random from the $1 / 3$-octaves from the range $100-2000$ $\mathrm{Hz}$. The subjects were asked to point to the brighter signal. The spectral content of the multitones, i.e. difference in harmonic number $\Delta_{H}$, and their duration $T D$ for different levels of difficulty are presented in Table IX. In the first part of the task (difficulty levels, $n=1 \div-5$ ), the difference in the number of harmonics in dark and bright sound was the same, e.g. at the difficulty level 2 , the multitones to be compared had two (dark sound) and six (bright sound) harmonics. At the difficulty level 6 , the number of harmonics was constant, the dark sound contained harmonics $2,3,4$, and 5 , while the bright sound contained harmonics 2, 4, 5 and 6 . At the difficulty levels 7 and 8, the number of harmonics was the same, while the signals differed in the amplitude of the highest component.

TABLE IX

Difference in harmonic number between dark and bright sound in the timbre discrimination of harmonic signals task.

\begin{tabular}{|c|c|c|c|c|c|c|c|c|}
\hline \multicolumn{9}{|c|}{ Timbre discrimination - harmonic signals } \\
\hline$n$ & 1 & 2 & 3 & 4 & 5 & 6 & 7 & 8 \\
\hline$\Delta_{H}$ & 6 & 4 & 2 & 1 & 1 & 0 & 0 & 0 \\
\hline$T D$ & 1 & 1 & 1 & 1 & 0.3 & 0.3 & 0.3 & 0.3 \\
\hline \multicolumn{9}{|c|}{ Harmonics — amplitudes } \\
\hline \multicolumn{3}{|c|}{$n$} & \multicolumn{2}{|c|}{6} & \multicolumn{2}{|c|}{7} & \multicolumn{2}{|c|}{8} \\
\hline \multicolumn{3}{|c|}{ Dark sound } & \multicolumn{2}{|c|}{$\begin{array}{l}f 2 f 3 f \\
4 f 5 f\end{array}$} & \multicolumn{2}{|c|}{$\begin{array}{l}f 2 f 4 f \\
0.5 \times 6 f\end{array}$} & \multicolumn{2}{|c|}{$\begin{array}{l}f 2 f 3 f \\
0.7 \times 4 f\end{array}$} \\
\hline \multicolumn{3}{|c|}{ Bright sound } & \multicolumn{2}{|c|}{$\begin{array}{l}f 2 f 4 f \\
5 f 6 f\end{array}$} & \multicolumn{2}{|c|}{$\begin{array}{l}f 2 f 4 f \\
6 f\end{array}$} & \multicolumn{2}{|c|}{$\begin{array}{l}f 2 f 4 f \\
3 f 4 f\end{array}$} \\
\hline
\end{tabular}


3.9. Lateralization of sound - tones (AT, part 1) and lateralization of sound - noise bands (AT, part 2)

The aim of both tasks was evaluation of sound image, resulting from sound lateralization. Pairs of tones/noise bands with different interaural intensity differences $(I I D)$ were presented. The subject compared two sounds of the same frequency in the case of tones and two $1 / 3$-octave noise bands having the same center frequency in the case of noise. The frequency range of tones/center frequencies of noise bands was 100-4000 Hz; frequencies were $1 / 3$-octave spaced. The signals in a pair differed in intensity. The subjects were asked to point out which signal in the pair comes from the right. Lower intensity level in the left channel corresponded to the sound image on the right-hand side. In each pair, the signal of $I I D=0 \mathrm{~dB}$ was always presented (perceived as coming at the angle $0^{\circ}$ according to the subject) as well as a signal IID of which depended on the task difficulty level. The aim was to point out the signal (first or second in the pair) which came from the right-hand side. IID between the left and right channel and duration of stimuli depending on the difficulty level are shown in Table X. Time intervals between signals were $1 \mathrm{~s}$ for difficulty levels $1-7$ and $1-6$ in the tasks with tones and noise bands, respectively. For the two last difficulty levels in both tasks, the time intervals were $500 \mathrm{~ms}$.

\section{TABLE X}

Signal parameters for lateralization of sounds task: tones and noise bands tasks.

\begin{tabular}{l|c|c|c|c|c|c|c|c|c}
\hline \hline \multicolumn{10}{c}{ Tones } \\
\cline { 1 - 9 } IID [dB] & -1 & 2 & 3 & 4 & 5 & 6 & 7 & 8 & 9 \\
\hline$T D[\mathrm{~s}]$ & 1 & 1 & 1 & 1 & 1 & 1 & 1 & 1 & 0.3 \\
\hline \multicolumn{10}{c}{ Noise bands } \\
\hline$n$ & 1 & 2 & 3 & 4 & 5 & 6 & 7 & 8 & \\
\hline$I I D[\mathrm{~dB}]$ & -10 & -6 & -4 & -3 & -2 & -1 & -1 & -0.7 \\
\hline$T D[\mathrm{~s}]$ & 1 & 1 & 1 & 1 & 1 & 1 & 1 & 0.3
\end{tabular}

3.10. Miscellaneous (AT, parts 1 and 2)

The aim of the tasks was to relax the subjects. The tasks were not scored and the level of difficulty did not depend on previous answers. The subjects were asked to count the number of acoustic events. In variant ' $a$ ', the exact command was to count the number of cars the subject heard, while in variant ' $b$ ' the subject was prompted to count how many times the dog had barked. In the second task, the subjects listened to the sound of passing cars and were asked to identify the faster car.

\section{Pre- and post-test (verification test)}

The verification test was composed of 6 tasks checking the pitch discrimination, pitch and timbre categorization, pitch memory, lateralization of a stationary sound source, lateralization of a moving sound source, and lateralization of two moving sound sources. The tasks came from AT and experiments described in $[2,7]$. The sound material was presented at a level of $65 \mathrm{~dB}$ SPL. The test apparatus and commands were the same as those used in AT. The verification test took 25 minutes.

Pitch discrimination test. The subjects were exposed to pairs of sounds, with each sound composed of a tone of one of the three frequencies $(500 \mathrm{~Hz}, 750 \mathrm{~Hz}$, or $1000 \mathrm{~Hz}$ ) and a tone of a frequency different by $0.25 \%$. 30 pairs were presented in total, 10 of each frequency. The individual tone duration is $300 \mathrm{~ms}$ and the interval between them was $300 \mathrm{~ms}$, the time to respond was 4 seconds. The detuning by $0.25 \%$ corresponded to the frequency discrimination assumed in literature [24] at the detection threshold. It should be detectable by the persons taking part in the training.

Pitch and timbre categorization task. The listeners were asked to compare randomly chosen pairs from among four multitones in the test for which parameters, possible answers, instructions and commands were taken from [2]. When the sounds differed in pitch, the pairs of signals had different frequency and the same spectral content. When the sounds differed in timbre, the signals had the same fundamental frequency and different spectral contents. When the objective was to discriminate between both pitch and timbre, the signals in a given pair had different fundamental frequency and different number of harmonics. In total, 40 pairs of multitone signals were presented, 15 differing in frequency, 12 differing in spectral content, and 13 differing in both frequency and spectral content.

Pitch memory task. The task was similar to that described in the training part, (subsection 3.4), but in the version in the verification test, only the 20 sequences of 9 tones were presented.

Lateralization of a stationary sound source, lateralization of a moving sound source, lateralization of two moving sound sources. The mode of signal recording, their parameters, indications, instruction for the listeners and the way of noting the score came from [7]. The sound of a drum and the sound of passing cars were presented through headphones. The sound of a drum reached the listener at the angles $\theta=n \times 45^{\circ}, n=0,1, \ldots 7$ in the horizontal plane and from above the head (27 sound examples, 3 from each direction). The sounds of a passing car were presented to simulate the passage of one car going in two opposite directions in front of the listener, behind the listener, and on either side of the listener. Three signals for each path and each direction were presented. In the case of the sounds simulating the passage of two cars going in two opposite directions in front of the listener, behind the listener, and either side of the listener, 3 signals for each path and direction were presented.

\subsection{Preliminary verification of the auditory training}

The preliminary verification test was performed in order to check the correctness of difficulty levels adopted for AT and verification tests, check the correctness of technical implementation of the software, e.g. software stability and compilation and, point out possible corrections. 
The subjects taking part in the test were 20 persons in the age of 20-27 years, of normal hearing and sight, with no musical education. The subjects were divided into two groups, each of 10 subjects - one group took part in the training, and the other was the control group. The trained group also took part in the pre- and post-test. The control group took part only in the pre- and posttest, held at the interval equal to the time of AT duration. Subjects were paid for their services. The participants could withdraw from the experiment at any time but they did not.

Statistical analysis of results was carried out with the use of ANOVA method. No statistically significant differences in means were observed in the results of the preand post-training verification tests and between the control and the trained groups. The only exception was the task concerning lateralization of stationary sound sources for the trained group for which difference in means was statistically significant, $p=0.023296$. Detailed values of means $M$ and standard deviations $S D$ in trained and control groups are shown in Table XI. The reasons for the lack of positive effect of AT, except one task mentioned above, could be a few: tasks too difficult — in particular when the parameters reached the threshold values; criterion of approval too restrictive; the method of constant stimuli providing quick results but of small sensitivity; the age of persons taking part in the test and their motivation insufficient (sighted persons). Some suggestions that could help eliminate some of the above reasons are given in the conclusions.

TABLE XI

Percent of correct responses in the verification test, $n s d$ - no statistical difference, $s s$ - sound source.

\begin{tabular}{|c|c|c|c|c|}
\hline \multirow{2}{*}{ Task } & \multicolumn{2}{|c|}{ Trained group } & \multicolumn{2}{|c|}{ Control group } \\
\hline & pre-test & post-test & pre-test & post-test \\
\hline \multirow{3}{*}{$\begin{array}{l}\text { Pitch } \\
\text { discrimi- } \\
\text {-nation }\end{array}$} & $M=0.52$ & $M=0.51$ & $M=0.50$ & $M=0.49$ \\
\hline & $S D=0.17$ & $S D=0.12$ & $S D=0.14$ & $S D=0.13$ \\
\hline & \multicolumn{2}{|c|}{$n s d$} & \multicolumn{2}{|c|}{$n s d$} \\
\hline \multirow{3}{*}{$\begin{array}{l}\text { Pitch/timbre } \\
\text { categori- } \\
\text { zation }\end{array}$} & $M=0.41$ & $M=0.48$ & $M=0.55$ & $M=0.57$ \\
\hline & $S D=0.18$ & $S D=0.23$ & $S D=0.24$ & $S D=0.24$ \\
\hline & \multicolumn{2}{|c|}{$n s d$} & \multicolumn{2}{|c|}{ nsd } \\
\hline \multirow{3}{*}{$\begin{array}{l}\text { Pitch } \\
\text { memory }\end{array}$} & $M=0.63$ & $M=0.68$ & $M=0.69$ & $M=0.75$ \\
\hline & $S D=0.17$ & $S D=0.14$ & $S D=0.11$ & $S D=0.15$ \\
\hline & \multicolumn{2}{|c|}{$n s d$} & \multicolumn{2}{|c|}{$n s d$} \\
\hline \multirow{3}{*}{$\begin{array}{l}\text { Lateraliza- } \\
\text {-tion of sta- } \\
\text { tionary } s s\end{array}$} & $M=0.36$ & $M=0.46$ & $M=0.47$ & $M=0.52$ \\
\hline & $S D=0.33$ & $S D=0.33$ & $S D=0.37$ & $S D=0.36$ \\
\hline & \multicolumn{2}{|c|}{$p=0.023296$} & \multicolumn{2}{|c|}{$n s d$} \\
\hline \multirow{3}{*}{$\begin{array}{l}\text { Lateraliza- } \\
\text { tion of a } \\
\text { moving } s s\end{array}$} & $M=0.69$ & $M=0.65$ & $M=0.68$ & $M=0.65$ \\
\hline & $S D=0.34$ & $S D=0.37$ & $S D=0.35$ & $S D=0.35$ \\
\hline & \multicolumn{2}{|c|}{$n s d$} & \multicolumn{2}{|c|}{ nsd } \\
\hline \multirow{3}{*}{$\begin{array}{l}\text { Lateraliza- } \\
\text { tion of two } \\
\text { moving } s s\end{array}$} & $M=0.65$ & $M=0.65$ & $M=0.71$ & $M=0.77$ \\
\hline & $S D=0.32$ & $S D=0.37$ & $S D=0.36$ & $S D=0.30$ \\
\hline & \multicolumn{2}{|c|}{$n s d$} & \multicolumn{2}{|c|}{$n s d$} \\
\hline
\end{tabular}

\section{Conclusions}

Analysis of the above-presented results has allowed to draw the following conclusions:

1. the auditory training did not bring the expected results in the group of adult persons of good sight taking part in the test;

2. the measures suggested for the future include: an increase in the number of persons taking part in the study; decrease of the threshold of approval from $90 \%$ to $70 \%$ of correct answers allowing quicker accomplishment of subsequent stages of AT and counteracting weariness and discouragement of subjects; the use of the adaptation method in the verification test task on discrimination of the pitch; increase of the number of stimuli presented in tasks $4-6$ on lateralization of the sound source in the verification test.

\section{Acknowledgments}

The work was supported by the National Science Center (Grant no. 2012/05/B/HS6/03863).

\section{References}

[1] E. Bogusz, E. Skrodzka, E. Hojan, M. Jakubowski, A. Talukder, D. Hojan-Jezierska, Pol. J. Environm. Stud. 20, 1395 (2011).

[2] E. Bogusz, H. Koprowska, E. Skrodzka, Acta Phys. Pol. A 121, A19 (2012).

[3] E. Hojan, M. Jakubowski, A. Talukder, H. Wereda, A. Furmann, R. Ewertowski, E. Skrodzka, P. Perz, P. Pękala, E. Bogusz, H. Lubawy, F. Tomaszewski, B. Czechyra, M. Orczyk, G. Szymański, M. Niewiarowicz, D. Hojan-Jezierska, A. Jezierska, Acta Phys. Pol. A 121, A5 (2012).

[4] B. Czechyra, E. Skrodzka, G. Szymański, F. Tomaszewski, J. Wiciak, Pol. J. Environm. Stud. 20, 45 (2011).

[6] E. Bogusz, H. Koprowska, E. Skrodzka, Archiv. Acoust. 37, 549 (2012).

[7] A. Furmann, E. Skrodzka, P. Giżewski, Ł. Nowotny, Acta Phys. Pol. A 123, 988 (2013).

[8] M. Pankowska, D. Hojan-Jezierska, E. Skrodzka, E. Szymiec, L. Kubisz, T. Świdziński, A. Wicher, Acta Phys. Pol. A 119, 131 (2011).

[9] J. Wiciak, B. Borkowski, D. Czopek, Acta Phys. Pol. A 123, 1101 (2013).

[10] J. Wiciak, B. Borkowski, I. Czajka, Acta Phys. Pol. A 121, A24 (2012).

[11] N. Lessard, M. Pare, F. Lepore, M. Lassonde, Nature 395, 278 (1998).

[12] B. Roder, F. Rosler, H.J. Neville, Neuroscience Letters 264, 53 (1999).

[13] K. Hugdahl, M. Ek, F. Takio, T. Rintee, J. Tuomainen, C. Haarala, Cognitive Brain Research 19, 28 (2004).

[14] A. Amedi, N. Raz, P. Pianka, R. Malach, E. Zohary, Nature Neurosci 6, 758 (2003). 
[15] B. Roder, F. Rosler, Int. J. Psychophysiol 50, 27 (2003).

[16] C.Y. Wan, A.G. Wood, D.C. Reutens, S.J. Wilson, Neuropsychologia 48, 344 (2010).

[17] P. Voss, F. Gougoux, R. Zatorre, M. Lassonde, F. Lepore, Neuroimage 40, 746 (2008).

[18] R.D. Easton, B.L. Bentzen J. Vis. Impair. Blind 93 (7), 405 (1999).

[19] K. Fiehler, J. Reuschel, F. Rosler, Neuropsychologia 47, 897 (2009).

[20] S. Gordon-Salant, S.A. Friedman, J. Speech. Lang. Hear. Res. 54, 622 (2011).
[21] H. Burton, J.B. Diamond, K.B. McDermott, J. Neurophysiol. 90, 1965 (2003).

[22] H. Burton, R.J. Sinclair, D.G. McLaren, Human Brain Mapping 23, 210 (2004).

[23] S. Roffler, R. Buttler, J. Acoust. Soc. Am 43, 1260 (1968).

[24] B.C.J. Moore, An introduction to the Psychology of Hearing, 1st edition, PWN, Poznań 1999 (in Polish). 